SCHWERPUNKT

\section{Mobilfunk - Risikodiskurse in Wissenschaft, Politik und Öffentlichkeit}

\author{
Einführung in den \\ Schwerpunkt
}

\author{
von Christoph Revermann, ITAS / TAB
}

Nach wie vor dominiert häufig der Konjunktiv die Diskussionen über die Auswirkungen hochfrequenter elektromagnetischer Felder (EMF) der Mobilfunktechnik. Der vorliegende Schwerpunkt versucht vor dem Hintergrund des wissenschaftlichen Kenntnisstandes zu möglichen gesundheitlichen Risiken des Mobilfunks eine knappe Darstellung der resultierenden Diskurse. Er skizziert Dissens und Konsens bei der Beurteilung gesundheitlicher Gefährdungen sowie sich hierauf beziehende mögliche Maßnahmen zur Risikominderung. Darüber hinaus werden die öffentliche Debatte bzw. die in Teilen der Öffentlichkeit geführte Risikokommunikation zum Thema EMF, die Rolle der Medien, der Medizin, der Netzbetreiber und der Länder (und Kommunen) sowie die gesellschaftlich relevanten und möglichen sozialen und ethischen Fragen angesprochen. Die leitende Fragestellung ist die nach der "Ursache“ und / oder „Lösbarkeit“ der offensichtlichen Diskrepanz einer intensiv genutzten Technologie (hoher Durchdringungsgrad) und ihres zugleich hohen Problematisierungsgrades in (Teilen) der Gesellschaft.

\section{Thematischer Hintergrund}

Mobiltelefone stellen weltweit ein nicht mehr wegzudenkendens Instrument der modernen privaten und beruflichen Kommunikation dar. International wächst der Markt rapide, weltweit werden bis Ende des Jahrzehnts drei Milliarden Mobilfunknutzer prognostiziert. In sehr vielen Ländern gibt es mindestens eine vollständige
Versorgung der Bevölkerung mit Mobiltelefonen (z. B. fast 100 Mio. Mobiltelefone in Deutschland). Der Mobilfunk ist zudem zu einem wichtigen Wirtschaftsfaktor und einer hoch innovativen Branche mit kurzen Innovationszyklen geworden. Die mittel- oder unmittelbar mit mobilen Funktechnologien befassten Unternehmen beschäftigen allein in Deutschland über 200.000 Menschen.

\subsection{Das Problem: Besorgnis der Öffentlichkeit}

In den vergangenen Jahren sind EMF-Quellen zum Teil Gegenstand kontroverser Diskussionen in der Öffentlichkeit und auch in der Wissenschaft geworden. Teile der Bevölkerung befürchten, dass EMF für Menschen negative gesundheitliche Folgen haben könnten (Wiedemann, Schütz 2002). Betroffene berichten subjektiv immer wieder von gesundheitlichen Beeinträchtigungen und Krankheitssymptomen, die sie mit hochfrequenten EMF in Verbindung bringen. Im Zentrum der (öffentlichen) Kontroversen um EMF stehen die Mobiltelefone und die zu deren Nutzung notwendigen Sendeanlagen als Träger hochfrequenter EMF. Die Besorgnis hinsichtlich der Mobilfunkstrahlung ist einerseits durch die flächendeckende Verbreitung dieser Technik begründet (fast jeder ist betroffen), sie lässt sich aber auch auf den Umstand zurückführen, dass Mobiltelefone zumeist in unmittelbarer Nähe zu empfindlichen Körperteilen (Gehör, Auge, Gehirn) genutzt werden. Andererseits müssen die Emissionen, die von Sendeanlagen ausgehen, auch von denen ,getragen" werden, die selbst keine Mobiltelefone benutzen; über die Nutzung eines Mobiltelefons kann ggf. jeder selbst entscheiden, im Falle der Basisstationen bzw. Sendeanlagen hat der Einzelne jedoch weniger Möglichkeiten, sich der Exposition zu entziehen.

Obwohl die Wirkungen von $\mathrm{EMF}^{1}$ auf den Menschen schon seit vielen Jahren Gegenstand wissenschaftlicher Untersuchungen sind und es eine fast unüberschaubare große Zahl (bis heute über 30.000 Publikationen) ${ }^{2}$ von (z. T. divergierenden) Forschungsergebnissen gibt, scheint man nach wie vor nicht zu endgültigen bzw. eindeutigen Aussagen über eventuelle Beeinträchtigungen der Gesundheit durch EMF kommen zu können. Als sich der Mobilfunk vor gut 
zehn Jahren als Massentechnik etablierte, nahm die mit unabhängigen Wissenschaftlern besetzte internationale Kommission zum Schutz vor nicht-ionisierender Strahlung (ICNIRP) eine kritische Sichtung der bis dahin durchgeführten wissenschaftlichen Untersuchungen vor. Die seitdem und bis dato gesicherten wissenschaftlichen Befunde zu biologischen Wirkungsschwel$\operatorname{len}^{3}$ von hochfrequenten EMF bilden die Grundlage für die Grenzwertempfehlungen der ICNIRP. Diese Grenzwerte liegen um ein Vielfaches unter den ermittelten kritischen Werten. Nationale und internationale Expertengruppen und Institutionen, wie die Weltgesundheitsorganisation (WHO), die ICNIRP, die Strahlenschutzkommission (SSK) und das Bundesamt für Strahlenschutz (BfS) in Deutschland, beurteilen die Grenzwertfrage bislang so, dass es nach ihrer Einschätzung beim derzeitigen Forschungsstand keine wissenschaftlich gesicherten Hinweise darauf gibt, dass bei Einhaltung der geltenden Grenzwerte Gesundheitsrisiken durch EMF bestehen. ${ }^{4}$ Alle begutachteten und replizierten Analysen ergaben, dass negative gesundheitliche Wirkungen nur dann (und mit einer Eventualität verbunden) auftreten können, wenn die absorbierte Strahlung das Körpergewebe zu stark erwärmen würde (BfS 2008; FDA 2008; WHO 2006).

Zum Teil blieben dennoch Zweifel, ob es neben der in den Grenzwerten berücksichtigten thermischen Wirkung auch nicht-thermische Wirkungen geben könnte, die möglicherweise schon bei Feldstärken zu Wirkungen oder Schäden führen könnten, die unterhalb der Grenzwerte auftreten. So werden u. a. Migräne und Kopfschmerzen, Schlaf- und Konzentrations- sowie allgemeine Befindlichkeitsstörungen häufig in einen Zusammenhang mit diesen „athermischen“ Effekten der EMF gebracht. Einerseits weisen Kritiker zwar darauf hin, dass sich die Effekte schwacher Strahlung bislang nicht replizieren ließen. Andererseits kann jedoch nicht zweifelsfrei erwartet werden, dass athermische Effekte so stabil sind wie thermische Effekte. Ebenso kann bei athermischen Effekten nicht erwartet werden, dass exponierte Personen in gleicher Weise auf die schwache Strahlung reagieren, da die fraglichen möglichen Wirkungen in einem sehr engen Zusammenhang mit elektrochemischen Prozessen des menschlichen Körpers stehen. So wird - von manchen - vermutet, dass ein kleiner Teil der Bevölkerung ,elektrosensibler“ sein könnte als die überwiegende Mehrheit.

Anfängliche (politische) Reaktionen zielten häufig darauf ab, die Öffentlichkeit davon $\mathrm{zu}$ überzeugen, dass ein Risiko nicht besteht, unerheblich oder zumindest viel geringer ist, als andere (alltägliche) Risiken. In der Regel stellten jedoch die zuständigen Stellen bald fest, dass es unabdingbar ist, sich der von Teilen der Öffentlichkeit vorgetragenen Bedenken konstruktiv anzunehmen sowie ggf. resultierende Maßnahmen und Aufgaben zeitnah und effektiv durchzuführen und auch entsprechend zu kommunizieren. Ursache für die Skepsis in der Bevölkerung gegenüber EMF sind nicht nur die Fragen der biologischen Wirksamkeit hochfrequenter elektromagnetischer Strahlung, sondern auch, dass der Netzauf- und -umbau (vom GSM-Standard zum UMTS-Netz) ohne ausreichende Koordination der Betreiber untereinander erfolgte und von einer nicht immer und überall offensiven bzw. transparenten Informationspolitik begleitet wurde.

\subsection{Regulatorische Rahmenbedingungen und Selbstverpflichtungen}

$\mathrm{Zu}$ Beginn des Jahrzehnts hatte somit die Standortsuche für Sendeanlagen vor allem deshalb an Bedeutung gewonnen, weil die Mehrzahl der Bevölkerung sich aus den Entscheidungsprozessen bei der Standortsuche ausgeschlossen sah. Auch die Beteiligung der Kommunen an den Netzplanungen blieb hinter den Erwartungen zurück. Verwaltungen, Regierungen wie auch Mobilfunkfirmen befürchteten wegen der zunehmend auch von Kommunen unterstützten Einsprüche gegen Antennenstandorte ernsthafte Rückschläge beim Ausbau der Funknetze. Dies hätte nicht nur die Geschäfte der Letzteren schädigen, sondern auch die Wirtschaft insgesamt beeinträchtigen und die seinerzeit große Hoffnung auf eine starke Zunahme der direkt und indirekt vom Mobilfunk abhängigen Arbeitsplätze zunichte machen können. Um die Situation zu beruhigen, wurden auf verschiedenen Ebenen und von verschiedener Seite Vorschläge für konkrete Maßnahmen zur Vorsorgestärkung sowie $\mathrm{zu}$ mehr und besserer Kommunikation und Teilhabe (der Betroffenen) erarbeitet. 
Um dazu beizutragen, dass in der EU ein Grundkonsens zu Risikobewertung und -management innerhalb der Mitgliedsländer geschaffen wird, veröffentlichte der Rat der EU eine Empfehlung zur Begrenzung der Exposition der Bevölkerung gegenüber EMF $(0-300 \mathrm{GHz})^{5}$, und die Europäische Kommission im Jahr 2000 eine Mitteilung zur Anwendung des Vorsorgeprinzips. ${ }^{6}$ In Deutschland wurden seit 2001 seitens der Bundesregierung, des Deutschen Bundestages, der Mobilfunknetzbetreiber sowie der kommunalen Spitzenverbände erhebliche - auch finanzielle - Anstrengungen und Initiativen im Kontext der EMF-Debatte unternommen. Eine Selbstverpflichtung der Mobilfunkanbieter, eine Kooperationsvereinbarung zwischen Kommunen und Betreibern sowie das Aktionsprogramm der Bundesregierung bildeten ein Maßnahmenpaket, das auf „Freiwilligkeit und Selbstregulierung" setzte (vgl. Difu 2008). Besondere Eckpunkte stellten dabei die Beteiligung der Kommunen bei der Standortsuche für Sendeanlagen (konzertierte Funknetz- und Standortplanung), Aufklärung und Einbezug der Bevölkerung, Informationskampagnen, Messaktionen, EMFKataster sowie weitere umfangreiche Forschungsförderung zur Risikoabschätzung, Risikokommunikation und zur Anwendbarkeit der Erkenntnisse im Strahlenschutz dar. Auch die Ergebnisse eines vom Ausschuss für Bildung, Forschung und Technikfolgenabschätzung des Deutschen Bundestages angeregten TA-Projekts zu Mobilfunk aus dem Jahr 2003 hatten hierfür Relevanz (vgl. Revermann 2003). National von besonderer Bedeutung war die Initiierung des Deutschen Mobilfunk-Forschungsprogramms (DMF) durch das Bundesumweltministerium, die auf Empfehlung der Strahlenschutzkommission sowie des Sachverständigenrates für Umweltfragen erfolgte.

\subsection{Das Deutsche Mobilfunk- Forschungsprogramm}

Ausgehend u. a. von der Intention, nach Möglichkeit zukünftig ,alle Fragen“" nach den Einflüssen des Mobilfunks auf die Gesundheit möglichst überzeugend zu beantworten, sollte das DMF versuchen, systematisch das Grundlagenwissen zu vertiefen und zugleich vorhandenen Verdachtsmomenten (einer Gefährdung) nachzugehen. Im Rahmen des DMF, für dessen fachliche und administrative Umsetzung das BfS zuständig ist, wurden bis einschließlich 2008 über 50 Forschungsprojekte in den Bereichen Biologie, Epidemiologie, Dosimetrie und Risikokommunikation durchgeführt. Der Öffentlichkeit wurde eine unmittelbare Beteiligung an den Fachdiskussionen ermöglicht; alle Forschungsprojekte konnten im Internet eingesehen und dort sowie auf Fachveranstaltungen kommentiert werden. Im Sommer 2008 wurden im Rahmen einer vom BfS organisierten internationalen Veranstaltung die Ergebnisse des DMF sowie die Erfahrungen mit der Selbstverpflichtung der Mobilfunknetzbetreiber präsentiert und diskutiert (BfS 2008). Die Veranstaltung diente dazu, auch die durch das BfS und die SSK vorgenommenen Bewertungen der Forschungsergebnisse der Öffentlichkeit sowie Entscheidungsträgern vorzustellen. Ergebnisse und Fazit sollen zudem in die bevorstehende Aktualisierung der Risikobewertung hochfrequenter EMF durch die WHO Eingang finden, die voraussichtlich 2009 erfolgen soll.

\section{Risiko - Bewertung, Information und Kommunikation}

Mit dem Abschluss des deutschen Forschungsprogramms, aber auch anderer nationaler und internationaler Vorhaben, die bereits abgeschlossen sind oder 2009 abgeschlossen wer$\mathrm{den}^{7}$, sind zwar fast alle etwas klüger, aber noch nicht unbedingt wirklich glücklicher. Eine Bewertung und Umsetzung der Forschungsergebnisse ist - insbesondere für die Politik - nicht nur aufgrund der Materie schwierig, sondern auch, weil die Interessen, Vorstellungen, Wahrnehmungen, Befürchtungen und Risikoempfindungen der Bevölkerung sehr heterogen sind. $\mathrm{Zu}$ Beginn des Jahrzehnts gab es noch wenige bundesweite Untersuchungen über die allgemeine Risikowahrnehmung in der Gesellschaft, und eine systematische Analyse der gesellschaftlichen Wahrnehmung der Mobilfunkthematik schon gar nicht. Mittlerweile konnten sowohl die jährlich durchgeführten repräsentativen und deskriptiven Umfragen (z. B. infas), spezielle Analysen zur Risikokommunikation im Mobilfunk (Schütz 2008; Siegrist et al. 2005; Wiedemann, Schütz 2003; Wiedemann et al. 2003) als auch entsprechende DMF-Projekte verdeutlichen, dass das Ausmaß der öffentlichen Besorg- 
nis und der empfundenen gesundheitlichen Beeinträchtigungen durch Mobilfunk über die Jahre außerordentlich stabil ist: In bestimmten Bevölkerungsgruppen sind die Sorge bezüglich elektromagnetischer Felder und die subjektiv empfundene Beeinträchtigung - trotz aller Forschungen, Untersuchungen, Aufklärungen und allen Informationsangeboten - hoch, für den weitaus größten Teil der Bevölkerung spielen mögliche Auswirkungen von EMF keine Rolle. Den Bundesumweltminister führte diese Tatsache auf dem DMF-Abschlusskongress zu einem persönlichen Fazit: „Also, ich glaube nicht, dass wir mir dem, was wir nach sechs Jahren mit dem Kongress einem Zwischenstadium zuführen, gleichzeitig zur Befriedung der Diskussion in Deutschland, aber auch darüber hinaus, beigetragen haben" (Meyer 2008, S. 80). Einer der Gründe könnte sein, dass es nicht an der Menge, sondern an der Qualität der Informationen mangelt, die besser auf die unterschiedlichen Zielgruppen zugeschnitten werden müssen, um spezifische Bedürfnisse tatsächlich oder besser erfüllen zu können. Ein weiterer Grund könnte darin liegen, dass nicht kognitive Variablen, sondern affektive Wertungen die Risikowahrnehmung beeinflussen, wie Siegrist et al. (2006) in ihren Untersuchungen über implizite Einstellungen bei Laien und Experten belegen konnten.

Dass wissenschaftliche Laien Risiken i. d. R. nach anderen Kriterien beurteilen als Experten, konnte in verschiedenen Forschungsansätzen gezeigt werden. Während für Experten bei der Risikoabschätzung mögliche Schäden und Eintrittswahrscheinlichkeit von zentraler Bedeutung sind, beziehen Laien andere Aspekte in ihre intuitive Risikobeurteilung ein. Dazu gehören z. B. Kontrollierbarkeit, Freiwilligkeit der Risikoübernahme, persönliche Betroffenheit, Nutzen einer Risikoquelle oder Auswirkungen auf künftige Generationen (Wiedemann 2008). Laienerwartungen an Forschungsergebnisse liegt zudem häufig die folgende Einschätzung zugrunde: „Entweder es gibt ein Risiko, oder es gibt keins." Experten stellen Risikovergleiche an, Laien erwarten eindeutige „Ja/ Nein“-Bewertungen. Die auf Wahrscheinlichkeitsaussagen zurückgehenden Expertenansichten stoßen meist auf Unverständnis. Und in der Öffentlichkeit werden häufig einmalige Vorkommnisse als eindeutige Belege gewertet, wo die Wissenschaft auf Vergleichbarkeit und Wiederholbar- keit bestehen muss. In der Folge können kaum auflösbare Kommunikationsprobleme im Risikodiskurs auftreten, wenn sich wissenschaftlich differenzierte Bewertungen und emotional begründete Besorgnis gegenüberstehen. Die Anforderungen an Forschungsstrategien und experimentelle Methodik wie Exaktheit und Nachprüfbarkeit teilen Laien häufig nicht, sondern sie erwarten die Einbeziehung von Einzelfällen und persönlichen Erfahrungen. Letztlich ist somit die Diskussion um mögliche gesundheitsschädliche Wirkungen des Mobilfunks nicht nur von einem „Expertendilemma“ gekennzeichnet. Auch der manchmal geforderte „Null-Beweis“ ist nicht zu erbringen: Unbedenklichkeit ist nicht zu beweisen, immer nur die Schädlichkeit. Die beobachtbaren Kommunikationsprobleme führen schließlich im Risikodiskurs dazu, dass Wissenschaftler von Laien, insbesondere von Bürgerinitiativen und Vereinigungen Elektrosensibler, als parteiisch wahrgenommen werden, was die Verständigung außerordentlich erschwert - ein Problem, das bis dato nicht gelöst werden konnte.

Aus Sicht der Forscher und Wissenschaftler kann ein Weg aus dem „Expertendilemma“ nur über die Schaffung einer (noch) breiteren Wissensbasis durch weitere Forschung führen. Ihre Forderungen an das Management von Risiken lauten, die Forschung quantitativ (durch mehr Fördermittel) zu erweitern, an die Forschung hohe qualitative Anforderungen hinsichtlich Exaktheit, Nachprüfbarkeit und Wiederholbarkeit zu stellen sowie die Interdisziplinarität der Forschung (Medizin, Biologie, Elektrotechnik, Physik und Statistik) zu gewährleisten. Eine eindeutige Basis für eine Risiko-Nutzen-Abwägung kann jedoch aufgrund der Bewertungsunsicherheiten wohl nicht wirklich erwartet werden. Die Entscheidung über Bewertungsmaßstäbe und Bezugsgrößen bei der Risikobewertung bleibt weitgehend eine politische Aufgabe.

\section{Medienresonanz und das Engagement von Bürgerinitiativen}

In der Medienberichterstattung wurde Mobilfunk als möglicherweise gesundheitsschädlicher Einflussfaktor seit Beginn des Jahrzehnts immer stärker in den Mittelpunkt der öffentlichen Aufmerksamkeit gerückt. Die Resonanz in den Massenmedien verleiht einem Risiko 
eine gesellschaftliche und politische Relevanz. Das Thema Mobilfunk wird von den Medien relativ häufig aufgegriffen, da es bewährte Selektionskriterien erfüllt, wie z. B. den Bezug zu lokalen oder aktuellen Aktivitäten oder die Verbindung zu Reizthemen wie "Strahlung“, „Krebs" oder spezielle Gruppen (z. B. Kinder oder kranke Menschen). Einer zunächst vergleichsweise kleinen Zahl besorgter Menschen gelingt es hierbei häufig, relativ große Aufmerksamkeit zu erhalten.

Bei der Medienberichterstattung zu EMF beim Mobilfunk zeigen sich vielfach Anzeichen, dass die Häufigkeit, die ausgewählten Themen und die Auswahl der in den Berichten zu Wort kommenden Gruppen relativ deutlich auf Leserreaktionen abzielen. Jedoch wäre es zu einfach, direkt von Medieninhalten auf die Rezipienten zu schließen. Ein einfaches „Reiz-ReaktionsSchema" wird in der heutigen Medienwirkungsforschung kaum noch unterstellt. Vielmehr finden auch die Diskussion mit Peer Groups, die eigenen Erlebnisse oder bestimmte dauerhafte Werthaltungen Berücksichtigung bei Bewertungen des Einflusses von einzelnen Medienereignissen und ihrer Darstellung. Dennoch kann die Wirkung der Medien auf die Perzeption eines Risikos als hoch eingeschätzt werden, nicht jedoch als allein determinierende Einflussgröße. Dementsprechend zurückhaltend müssen die Wechselwirkungen zwischen Tendenzen in der Berichterstattung und ggf. Zunahme des Protests gegen hochfrequente EMF des Mobilfunks gewertet werden: Nehmen Proteste in der Bevölkerung zu, so führt dies in der Regel auch zu einer Ausweitung der Berichterstattung - wenngleich manchmal auch nur lokal oder regional. Insgesamt gesehen überwiegt die Berichterstattung über Konflikte. Zum Teil lassen sich in der Berichterstattung auch Formulierungen ausmachen, die auf einen unzureichenden Informationsstand der Journalisten zurückzuführen sind. Die Mehrzahl aller Meldungen befasst sich mit den Standorten für Funkantennen und den damit verbundenen Konflikten - insbesondere dann, wenn diese Konflikte durch Bürgerinitiativen in die Medien getragen werden.

Festzustellen ist, dass die Forderungen von lokalen Bürgerinitiativen und bundesweiten Vereinigungen gegen „Elektrosmog“ graduelle Unterschiede aufweisen. Größere Verbände (z. B. der BUND) verstehen ihre Aufga- be eher als Aufklärung für Bürgerinitiativen und versuchen, diese bei ihren Aktionen zu beraten und $\mathrm{zu}$ unterstützen. Dies reicht von Informationsmaterial bis hin $\mathrm{zu}$ personeller Unterstützung bei Anhörungen im Gemeinderat. Oftmals richten sie Maximalforderungen an Politik und Mobilfunkunternehmen; diese Forderungen zielen z. B. auf einen Stopp des Ausbaus der Mobilfunknetze, bis die Risiken geklärt sind, oder auf einen Rückbau vorhandener Antennenanlagen.

Diese Interessengruppen sehen die Freiwilligkeit bislang getroffener Vereinbarungen als unzuverlässig und unzureichend an und fordern eine gesetzliche Regelung. Aus ihrer Sicht hat die Beibehaltung der Grenzwerte in Deutschland zu einer „Verhärtung der Fronten“ beigetragen. Nach Einschätzung von Behörden und Mobilfunknetzbetreibern sind diese Vereinigungen in der Minderheit, werden jedoch von der Öffentlichkeit am stärksten wahrgenommen. Daneben existieren zahlreiche weitere Gruppen, die häufig lokal organisiert sind und denen es um ein noch weitergehendes und stärkeres Mitspracherecht bei der Standortsuche geht, als die bisherige Praxis aufgrund der Verbändevereinbarung gewährleistet. Sie fordern

- eine stärkere Beteiligung von Bürgern bzw. Kommunen an Standortverfahren und damit eine Demokratisierung des Netzausbaus, weil sie sich durch die von Behörden nicht im Einzelfall kontrollierte Standortwahl der Betreiber in ihren Anwohner- und Bürgerrechten verletzt fühlen;

- die Errichtung von Schutzzonen (z. B. keine Antennen in Wohngebieten, in der Nähe von Schulen, Kindergärten, Krankenhäusern oder Altenheimen);

- eine Senkung von Grenzwerten mit stärkerer Berücksichtigung des Vorsorgeprinzips (z. B. orientiert an den ,Schweizer Vorsorgewerten").

Für die EMF-Debatte im Mobilfunk ist somit zu konstatieren, dass (mittlerweile) alle gesellschaftlichen Gruppen (wie etwa zuständige Behörden, Betroffene und Bürgergruppen, Unternehmen, die Wissenschaft, die Umweltmedizin, die Medien sowie die Politik) in eine solche Arena fest und nachhaltig eingebunden sind. Während sich Medien, lokale Gruppen, Baubiologen, Umweltmediziner, die Scientific Com- 
munity sowie „Elektrosensible“ schon seit Längerem sehr stark engagieren, ist bei Netzbetreibern, Ärzteverbänden, Kommunen und der Öffentlichkeit größtenteils von einer wenig bis leicht interessierten Beteiligung zu sprechen, die allerdings in den letzten Jahren an Bedeutung und Einfluss gewonnen hat. Auch die Aktivitäten der Parlamente in Bund und Ländern sind spürbar angewachsen. Anhörungen und Debatten haben in Deutschland die Fachdiskussion beeinflusst und die breite Öffentlichkeit auf das Thema aufmerksam gemacht.

\section{Die Beiträge des Schwerpunktes}

Im ersten Beitrag gibt Gerd Friedrich einen zusammenfassenden Überblick zur Anwendungsvielfalt im Funkbereich. Einem kurzen Rückblick über die Entstehungsgeschichte des Einsatzes von Funkdiensten im öffentlichen und privaten Bereich folgt eine technische Erläuterung des aktuellen Mobilfunksystems sowie ein Ausblick auf die zukünftigen - und sich zum Teil schon in der ,pipeline“ befindlichen - Mobilfunkstandards der sogenannten vierten Generation, die in den kommenden Jahren die Übertragung höchster Datenraten bei höchster Geschwindigkeit adäquat ermöglichen sollen. Dies führt unweigerlich zu der Frage, wie sich aktuell die Situation der Exposition der Bevölkerung mit EMF darstellt und ob künftig mit einer weiter zunehmenden Belastung der Bevölkerung durch EMF zu rechnen ist. Perspektivisch postuliert der Autor eine Grundstrategie zum verantwortungsvollen Umgang mit technologischen Risiken am Beispiel der durch Funktechnologien entstehenden Emissionen bzw. ihrer Gesundheitsrelevanz: Grundsätzlich habe eine solche Bewertung frei von wirtschaftlichen und politischen Interessen zu sein. Nach seiner Einschätzung wird sich zum einen die Expositionslage der Bevölkerung nicht wesentlich verändern und sind zum anderen die bestehenden Vorsorgemaßnahmen - da auf umfassender Forschung sowie fundiertem wissenschaftlichen und medizinischen Wissen basierend - heute und auch beim zukünftigen Einsatz der neuen breitbandigen Funkanwendungen ausreichend.

Der Beitrag von Wolfgang Weiss, Rüdiger Matthes und dem Autor dieser Einführung be- fasst sich mit dem multidisziplinären Analyseund Erklärungsansatz des von 2002 bis 2008 durchgeführten Deutschen Mobilfunk-Forschungsprogramms (DMF) und gibt eine zusammenfassende Darstellung und Einordnung der Ergebnisse des DMF aus Sicht des zuständigen Bundesamtes für Strahlenschutz (BfS). Grundtenor ist, dass das DMF dazu beigetragen hat, die Erkenntnisse über mögliche gesundheitliche Risiken von hochfrequenten elektromagnetischen Feldern des Mobilfunks entscheidend zu verbessern, so dass insgesamt die bestehenden Grenzwerte bestätigt werden konnten. Die tatsächlichen Expositionen im Alltag sind wesentlich genauer bekannt als zu Beginn des DMF. Auch lieferten die Studien keine Hinweise auf bisher unbekannte Wirkungen. Auf der Basis dieser Ergebnisse scheint somit die Aussage berechtigt, dass, falls gesundheitliche Risiken der Felder des Mobilfunks unterhalb der Grenzwerte bestehen diese so gering sind, dass sie mit den derzeit zur Verfügung stehenden wissenschaftlichen Methoden qualitativ und quantitativ nicht ermittelt werden können. Folgende Themen haben zukünftig Forschungspriorität: zum einen mögliche Langzeitrisiken für Nutzungszeiten des Mobiltelefons von mehr als zehn Jahren, zum anderen die Frage, ob Kinder stärker durch hochfrequente EMF belastet sind bzw. ob sie empfindlicher reagieren als Erwachsene. Da hier Wissenslücken bestehen, hält das BfS weiterhin Maßnahmen der Vorsorge, die die Grenzwertregelungen ergänzen, für unabdingbar. Konstatiert wird jedoch auch, dass die aus wissenschaftlicher Sicht klaren Ergebnisse des DMF in der Öffentlichkeit zum Teil sehr unterschiedlich aufgenommen werden. Dies zeigt sich nach wie vor in dem hohen Maß an Aufklärungsbedarf, der formuliert wird. Will man nicht riskieren, dass die in Teilen der Bevölkerung vorhandene Besorgnis bestehen bleibt oder sich angesichts zukünftiger Entwicklungen weiterer Funktechnologien noch vergrößert, so ist dieser Aufklärungsbedarf auch zu „bedienen“. Deutlich wird, dass es eines dauerhaften Dialogs zwischen den Vertretern der Wissenschaft, der beteiligten Institutionen und Behörden und den Betroffenen bedarf - sicherlich ein langer und beschwerlicher Prozess, zu dem es allerdings auch keine Alternative gibt.

Andreas Kappos erläutert in seinem Beitrag, welche wichtigsten Aspekte mit welchen 
Gründen sich für die Ärzteschaft im Umgang mit eventuellen gesundheitlichen Risiken aus dem umfassenden Einsatz des Mobilfunks und der daraus resultierenden exponentiellen $\mathrm{Zu}-$ nahme der Exposition der gesamten Bevölkerung mit hochfrequenten EMF ergeben. Trotz der aktuellen wissenschaftlichen Erkenntnisse auf Grundlage vieler Forschungen und Forschungsprogramme bleibt aus ärztlicher Sicht die Frage nach möglichen athermischen Wechselwirkungsmodellen, die Frage nach individuell spezifischer Elektrosensibilität bestimmter Personen oder Personengruppen und die Möglichkeit von Langzeitwirkungen weitgehend ungeklärt. Ein wichtiger Grund ist, dass epidemiologische Untersuchungen, die zur Klärung z. B. der Frage der Kanzerogenität elektromagnetischer Felder aus dem Mobilfunk beim Menschen beitragen könnten, auf häufig unüberbrückbare methodische Schwierigkeiten stoßen - insbesondere bei der Bestimmung der individuellen Exposition. Daraus ergeben sich präventionsmedizinische Notwendigkeiten, und im Blick auf eine möglicherweise lebenslange Exposition mit hochfrequenten EMF mahnt Kappos zur Vorsicht und Vorsorge: $\mathrm{Zu}$ fordern sei, alle technischen Möglichkeiten zu nutzen und organisatorischen Maßnahmen $\mathrm{zu}$ treffen, um die Emission von EMF im Umfeld des Menschen zu minimieren, und zwar immer dann und solange, wie die Unbedenklichkeit der Exposition nicht evident ist. Der zweite Aspekt der ärztlichen Beschäftigung mit gesundheitlichen Wirkungen elektromagnetischer Strahlung ist der klinisch-kurative. Wie ist aber mit Personen umzugehen, die angeben, unter Einwirkung hochfrequenter elektromagnetischer Felder zu leiden und die über die unterschiedlichsten Beeinträchtigungen und Gesundheitsstörungen klagen, wenn sie entsprechend exponiert sind? Der Autor räumt ein, dass sich ein prinzipielles Risikokommunikationsproblem zwischen Arzt und Patient aus dem Sachverhalt ergeben kann, dass zwar schädliche Wirkungen hochfrequenter elektromagnetischer Strahlung wissenschaftlich nicht belegt sind, aber gleichzeitig zu vorsichtigem Verhalten beim Umgang mit entsprechenden Geräten geraten werden muss. In jedem Fall ist es eine Wanderung auf einem schmalen Grad zwischen Sorglosigkeit und Besorgnis.

Der Beitrag von Axel Böttger beschäftigt sich mit einem ganzen Bündel an Problemstel- lungen und Handlungsnotwendigkeiten, mit denen sich die Politik und die Bundesregierung seit der bundesweiten Einführung des digitalen Mobilfunks zu Beginn der 1990er Jahre konfrontiert sah. Seinerzeit war natürlich seitens der Industrie, aber auch in der Bevölkerung eine erwartungsvolle Begeisterung vorhanden, zugleich machte sich aber in bestimmten Bevölkerungsgruppen Skepsis gegenüber der für sie nicht einschätzbaren Technik und Befürchtungen vor gesundheitlichen Beeinträchtigungen bemerkbar. Die Politik reagierte relativ umgehend mit einer gesetzlichen Regelung zu EMF: der Bundesimmissionsschutzverordnung. Da auf dem Höhepunkt des Mobilfunknetzausbaus Ende der 1990er Jahre jedoch von einigen Staaten Europas „Vorsorgegrenzwerte“ eingeführt wurden, hielt der gesellschaftliche Druck auf die Regierungsstellen an. Dies führte schließlich erstens zu einer Art „freiwilligen Verpflichtung“ der Mobilfunkbetreiber zu dauerhaften, nachprüfbaren Verbesserungen in den Bereichen Verbraucher-, Gesundheits- und Umweltschutz sowie zu einer intensiven Zusammenarbeit mit allen Kommunen bei der Standortplanung für Sendeanlagen. Zweitens wurde vom Bundesumweltministerium das DMF auf den Weg gebracht, denn im Zuge des massiven Netzausbaus hatte sich die Konfliktsituation in den Gemeinden und mit betroffenen Menschen keineswegs geändert. Mittlerweile kann jedoch als eine Bilanz aus den kontinuierlichen Maßnahmen konstatiert werden, dass die Zahl der Konflikte sowohl absolut als auch relativ enorm abgenommen hat: Sie hat sich seit 2002 halbiert. Insgesamt bewertet der Autor - stellvertretend für die Bundesregierung - die ergriffenen Maßnahmen zur Kommunikation und Partizipation, zum Verbraucherschutz, zur Forschung, zum Monitoring und Aufbau von Informations- und Datenbanken sowie zum Risikomanagement insgesamt als erfolgreich und die wesentlichen Ziele als erreicht. Die Notwendigkeit zum kontinuierlichen Dialog bleibt jedoch.

Die Aktivitäten bezüglich des weiteren Netzaus- und -umbaus des Mobilfunks sowie die damit verbundenen Notwendigkeiten, Probleme und Konflikte in den einzelnen Bundesländern und den jeweiligen Kommunen - in all ihrer Unterschiedlichkeit und Vielfalt - sind Thema des Beitrags von Johannes Grützner. Bei Gemeinden, besorgten Einwohnern und bei 
Mobilfunkkritikern stehen die sichtbaren Auswirkungen dieses Ausbaus - die MobilfunkSendeanlagen - besonders im Blickpunkt und die Kommunen häufig vor einem Dilemma: Es galt (und gilt) einerseits, die Wettbewerbsfähigkeit einer Kommune oder Region zu erhalten oder zu verbessern, also zu vermeiden, dass gewerbliche wie private Kunden, über fehlende Netzabdeckung und schlechte Erreichbarkeit klagen. Gleichzeitig mussten Forderungen Betroffener „befriedet“" werden, den Mobilfunkausbau durch die zuständigen Behörden oder die Gemeinde zu stoppen, solange nicht alle Fragen den Schutz vor elektromagnetischen Feldern betreffend geklärt seien. Noch Anfang des Jahrzehnts erhielt eine Kommune in aller Regel kaum Möglichkeiten, ihre Interessen frühzeitig einzubringen. Um bei diesen Entscheidungen aber Einfluss nehmen zu können, musste bereits bei der Netzplanung mitgewirkt werden. Entsprechende Forderungen wurden von betroffenen Städten und Gemeinden sowie den Ländern - auch auf politischer Ebene - gegenüber Bundesregierung und Wirtschaft vehement geäußert. In der Folge veranlassten auch diese Problematisierungen und Forderungen auf Länderebene letztlich den Bund zur Erarbeitung rechtlicher Regelungen, auch brachten die o. g. Selbstverpflichtung der Mobilfunkbetreiber und die weiteren Maßnahmenpakete der Bundesregierung hier Fortschritte. Der Autor konstatiert aber auch aktuelle Defizite: Insbesondere kleinere und ländliche Kommunen, in denen Mobilfunk aufgrund der geringen Anzahl von Sendeanlagen im Alltagsgeschäft eher ein „Randthema“ ist, benötigen praktische Unterstützung hinsichtlich der Rolle, die ihnen im Rahmen der Selbstverpflichtung zugeschrieben wurde, und der Frage, wie sie mit den Besorgnissen der Bevölkerung durch eine geeignete Risikokommunikation umgehen.

Thomas Eikmann und Caroline Herr stellen anhand des Beispiels einer einzigen Medienkontroverse über die Bedeutung der Ergebnisse einer einzigen wissenschaftlichen Publikation exemplarisch dar, welchen Einfluss Medien auf die Meinungsbildung oder Meinungsführerschaft im Kontext eines spezifischen Problembereichs erlangen können. Dies ist insofern sowohl interessant als auch problematisch, als von mehr als 90 Prozent der Bevölkerung die Medien als wichtigste Informationsquelle für die
Risiken des Mobilfunks angegeben werden. Die von den Autoren analysierte Medienkontroverse zeigt in all seinen Facetten sowohl die Problematik der Berichterstattung von wissenschaftlich komplexen Tatbeständen, als auch deren Bewertung in den Medien. Die diskrepante Interpretation von Forschungsergebnissen, die im wissenschaftlichen Diskurs als „,normal“ und sogar als erforderlich einzustufen ist, führt in der Öffentlichkeit dagegen häufig zu Verunsicherung und zur Bestätigung von (Vor-)Urteilen. Gleichzeitig demonstriert das Beispiel aber auch die Problematik der (vorzeitigen) Publikation von Forschungsergebnissen in den Medien sowie die sich auch daraus ergebenden (schwierigen) Beziehungen zwischen Wissenschaftlern und Medienvertretern. Darüber hinaus wird die bedeutende Rolle der Medien im Aktionsbereich der verschiedenen Akteure im Bereich Mobilfunk deutlich. Und nicht zuletzt wird - bei vermeintlich unklarer wissenschaftlicher Datenlage herausgestellt, dass persönliche Überzeugungen die Berichterstattung teilweise vielleicht stärker bestimmen als in anderen Problembereichen. Medienkontroversen in derart sensiblen Informationsbereichen können daher leicht zu Verunsicherung der Leser und letztendlich auch zu Zweifeln an der Vertrauenswürdigkeit der Berichterstattung führen.

Abschließend diskutiert Christiane Pölzl, welche grundlegenden Erkenntnisse zur Informationsaufnahme und -verarbeitung sowie zur Risikowahrnehmung aus der Forschung bekannt sind. Sie stellt die Frage nach den besonderen Aspekten und Herausforderungen in der Bewertung und Kommunikation der Ergebnisse des DMF für das Bundesamt für Strahlenschutz (als federführender Institution). Die Autorin verdeutlicht, dass neben empirischen Erkenntnissen die Wahrnehmung und Verarbeitung von wissenschaftlichen Informationen in der Öffentlichkeit einen wesentlichen Einfluss auf den Kommunikationsprozess besitzen, in dem die jeweiligen Bewertungen der Risiken von Wissenschaft und Bevölkerung konfligieren. Zusammengefasst ist nach ihrer Ansicht zu konstatieren, dass die durch viele Akteure vertretene Annahme, es bestünde erstens ein hohes Informationsbedürfnis in der Bevölkerung zu EMF und zweitens wäre eine hinreichende Bereitstellung an Informationen ausreichend, um ein adäquates Wissen in der Bevölkerung zu erreichen, kritisch hinter- 
fragt werden muss. In der Realität ist bei einem Großteil der Bevölkerung die tatsächliche Bereitschaft gering, sich mit technisch und wissenschaftlich komplexen Themen auseinanderzusetzen. Die sich daraus ergebende schwierige Situation ist eine der zentralen Herausforderungen für die Risikokommunikation. Eine mögliche Lösung, einem unterschiedlichen Informations- und Kommunikationsverhalten in der Bevölkerung gerecht zu werden, besteht aus Sicht des BfS in einem kontinuierlichen Austausch mit Multiplikatoren und Stakeholdern, die jeweils zielgruppenspezifische Ansprechpartner für die verschiedenen Bevölkerungsgruppen sind. Die geplante Fortführung des „Runden Tisches zum Deutschen Mobilfunk-Forschungsprogramm“ zu Themen der neuen Funktechnologien könnte eine weitere geeignete Plattform für einen entsprechenden Informationsaustausch und Risikodiskurs bieten.

\section{Schlussbetrachtung}

In den letzten Jahrzehnten werden eine ganze Reihe von Systeminnovationen von Risikokontroversen begleitet. Kernenergie, Gentechnik, Klimakatastrophe und Elektrosmog sind Beispiele für Risikothemen, die nicht nur unter Experten, sondern auch in der Öffentlichkeit und den Massenmedien diskutiert werden. Die Debatten entwickeln sich dabei zu einem Strukturmerkmal moderner Gesellschaften, in denen die Entscheidung über die Einführung neuer Technologien immer auch mit Fragen der Risikobewertung, der Akzeptabilität und der Regulierung einhergeht. In diesem Zusammenhang ist es ein Verdienst der Risikokommunikationsforschung (z. B. Baumgärtner 2005; Bechmann, Stehr 2000; Bentele et al. 2003; Petermann 2001; Renn et al. 2007; Wiedemann, Schütz 2008), darauf hingewiesen zu haben, dass diese Debatten sinnvolle und diskursive Auseinandersetzungen über die Bestimmung und Ausgestaltung des gesellschaftlichen Nutzungsverhaltens darstellen und nicht etwa „Störfälle“.

Die Tatsache, dass ein Risikodiskurs stattfindet, darf jedoch nicht mit der Erwartung verbunden werden, eine solche Auseinandersetzung führe mit einem gewissen Automatismus mittel- oder langfristig zu einem Konsens über die Risikobewertung und die zu treffenden
Maßnahmen. Eine solche Erwartungshaltung erscheint schon deshalb problematisch, weil Risikoeinschätzungen $\mathrm{zu}$ einem wesentlichen Teil von divergierenden Leitbildern und Werten bestimmt werden. Risikokommunikation bietet hiernach nicht zuerst die Chance der Lösung eines Konflikts, sondern ist als Versuch $\mathrm{zu}$ werten, auch bei scheinbar unüberwindbaren Dissensen und Interessenlagen zu möglichst vielseitig akzeptierten Kompromisslösungen zu gelangen.

Gleichwohl können natürlich aus dem wissenschaftlichen Gesamtbild der Risikoeinschätzung Schlussfolgerungen für eine Risikobewertung gezogen werden. Dies geschieht jedoch nach wie vor - auch unter Unsicherheit, beispielsweise im Hinblick auf die Frage, ob und ggf. welche Maßnahmen des Risikomanagements zu einer Vorsorge zu tätigen sind. In der einschlägigen Literatur zur Risikobewertung findet sich diesbezüglich eine Reihe von Klassifikationsvorschlägen, die teilweise auch in der Praxis Anwendung finden. Unterschieden wird beispielsweise zwischen ,wissenschaftlichem Nachweis“, „wissenschaftlich begründetem Verdacht" und „wissenschaftlichem Hinweis“.

Mit großer Wahrscheinlichkeit sind beim Thema Mobilfunk die wissenschaftlichen Kernfragen mittlerweile hinreichend beantwortet. Die wissenschaftlichen Grundlagen für die mögliche Identifizierung tatsächlicher gesundheitlicher (oder ökologischer) Risiken dürften ausreichend sein. Dies kann jedoch nicht ausschließlich dahingehend interpretiert werden, dass politische Entscheidungsträger sich allein auf die Forschung verlassen können. Angesichts der nach wie vor bestehenden Unsicherheit in Teilen der Bevölkerung bzw. bei sich von hochfrequenter Strahlung negativ betroffen Fühlender sollte der politische Maßnahmenkatalog ggf. breit(er) gefächert sein. Auf diesen Personenkreis bezogen sollte und kann vermutlich nicht auf nochmals weitere oder evtl. neue wissenschaftliche Erkenntnisse gewartet werden. Eher können fallbezogenes, konkretes und konstruktives Eingehen auf die (öffentlich) vorgetragenen Bedenken sowie die Einführung von - auch persönlichen und lokalen - Vorsorgemaßnahmen zielführend sein. Dies kann durch unterschiedliche Maßnahmen erfolgen (vgl. u. a. BfS 2008). Zu diesen Vorsorgemaßnahmen kann auch eine vorsorgliche Minimierung der Exposition gehö- 
ren: Beim Betrieb der bestehenden und der Entwicklung neuer drahtloser Kommunikationstechnologien wäre auf eine weitere Optimierung im Sinne des Strahlenschutzes zu achten. Im privaten Bereich kann die Wahl und Handhabung der relativ stärksten EMF-Quellen (u. a. Mobiltelefon, DECT-Basisstationen, Babyphone, WLAN) die Exposition erheblich verringern.

Kommunale Vertreter und die Fachleute der zuständigen Behörden können einen wichtigen Beitrag zu einer guten Risikokommunikation und den Umgang mit Standortkonflikten bei Mobilfunk-Antennen auf lokaler Ebene leisten. Hier kann beispielsweise der aktuell erstellte Mobilfunk-Ratgeber für Kommunen, insbesondere für kleinere und ländliche, Unterstützung bieten (http://www.ratgeber-mobilfunk.de). Eine dauerhaft wichtige Aufgabe aufseiten der Kommunen ist auch, die notwendigen sozialen und kommunikativen Kompetenzen für eine Verbesserung des Dialogs sowohl mit den Netzbetreibern, als auch mit den Bürgern aufzubauen und zu pflegen. Im Vordergrund steht dabei z. B. die frühzeitige Information der Bevölkerung über Standortplanungen, die Schaffung von Transparenz, das Eingehen auf Besorgnisse von Einwohnern und die Berücksichtigung ihrer Interessen soweit möglich. Beteiligte und Betroffene mit widersprüchlichen Interessen, an einen Tisch zu holen“, sollte als eine selbstverständliche Maßnahme zur Konfliktlösung angesehen werden. Sinnvoll kann das Hinzuziehen externer Fachkompetenz bei Diskussionen zu Risikound Gesundheitsthemen sein (vgl. http://www. bfs.de/de/bfs/druck/broschueren/bro dmf.html).

$\mathrm{Zu}$ bedenken ist auch, dass bei einer Risikobewertung unter dem Gesichtspunkt der Vorsorge zum einen das Problem (un)vollständiger Information besteht, zum anderen beim Risikomanagement in umfassender Weise die Interessen aller Beteiligten abgewogen werden müssen und drittens eine kritische Überprüfung der Zielerreichung durch die vorgeschlagenen Maßnahmen vorzunehmen ist. Und schließlich kommt bei der politischen Bewertung von Forschungsergebnissen (insbesondere) in Bezug auf die Festlegung von Grenzwerten den Parlamenten in Bund und Ländern eine besondere Aufgabe zu. Das Bundesverfassungsgericht hat in seinen Entscheidungen mehrfach diese Anforderung hervorgehoben. Die wissenschaftliche Begründbarkeit von Grenzwerten bildet dabei eine zentrale Anforderung für die Akzeptanz. Hinsichtlich weiterer Maßnahmen, die z. B. mit Blick auf das Vorsorgeprinzip eingeleitet werden (könnten), ist es eine bedeutende Aufgabe der parlamentarischen Debatte, eine für die Öffentlichkeit transparente Risiko-Nutzen-Abwägung im Rahmen bzw. im Vorfeld einer Entscheidungsfindung vorzunehmen.

Gleichzeitig müssen die verbleibenden wissenschaftlichen Unsicherheiten durch gezielte Forschungsanstrengungen weiter eingegrenzt werden. Dazu gehören insbesondere die Analyse von Wirkungen auf Kinder und die von Langzeitwirkungen. Noch offene Fragen betreffen weiterhin den Aspekt, welche Inhalte konkret dem Informationsbedürfnis verschiedener Zielgruppen entsprechen und auch von ihnen verarbeitet werden können. Zusätzliche Forschung ist für die Klärung der Frage erforderlich, wie wissenschaftliche Unsicherheiten gegenüber wissenschaftlichen Laien angemessen kommuniziert werden können. Das Problem besteht zum einen darin, dass ohne wissenschaftliches Grundverständnis Risikoinformationen nicht richtig verstanden werden können. Doch ohne dieses Wissen bleiben Informationen nutzlos oder verstärken gar Fehleinschätzungen. Weiterhin besteht die Schwierigkeit darin, dass Risikowahrnehmungen - einmal ausgebildet - weitgehend stabil sind. Sie lassen sich nicht ohne Weiteres verändern (Wiedemann 2008, S. 146). Vertiefte Erkenntnisse zur Entstehung von Besorgnis und der Rolle von Emotionen in der Risikowahrnehmung könnten daher zu einer weiteren Verbesserung der Risikokommunikation beitragen. Näher zu untersuchen ist auch die Rolle von Vertrauen und Glaubwürdigkeit für eine gute Risikokommunikation sowie die Möglichkeiten, darauf Einfluss zu nehmen (vgl. BfS 2008; Luhmann 1989; Schütz 2008).

Allgemeine Übereinstimmung besteht dahingehend, dass bessere Information eine zentrale Rolle in der Risikokommunikation über die Auswirkungen der Mobilfunktechnologie spielen sollte. Dabei darf nicht außer Acht gelassen werden, dass im Diskurs über hochfrequente EMF die Forschungsergebnisse immer wieder entsprechend aufbereitet und kommuniziert werden müssen, um von der Bevölkerung bzw. von den betroffenen Teilen der Bevölkerung rezipiert zu werden. Die Frage, wie Informationen neutral, differenziert und problemorientiert 
dargestellt, gebündelt und bewertet werden können, stellt eine Hauptanforderung für alle beteiligten öffentlichen Akteure dar. Zukünftige Informationsmaßnahmen für die Bevölkerung sollten klares Orientierungswissen bieten und mögliche Handlungsspielräume für den Einzelnen aufzeigen. Aussagen über wissenschaftliche Erkenntnisse und die Grenzen des Wissens müssen so einfach und konkret wie möglich formuliert werden. Nur so werden letztlich die komplexen Zusammenhänge in einer Risikokommunikation, aber auch deren Grenzen deutlich.

\section{Anmerkungen}

1) Unstrittig ist, dass elektromagnetische Wellen biologische Wirkungen verursachen können. Es muss jedoch klar zwischen einem „biologischen Effekt" und einer ,negativen gesundheitlichen Auswirkung" unterschieden werden. Obwohl die Literatur diese Termini nicht übereinstimmend benutzt, kann die folgende Unterscheidung mehr Klarheit verschaffen: Ein biologischer Effekt ist eine messbare (jedoch nicht notwendigerweise schädliche) physiologische Reaktion im biologischen System auf eine Exposition durch elektromagnetische Felder. Eine negative Auswirkung auf die Gesundheit ist ein biologischer Effekt, dessen Wirkungen (Folgen) über die normale physiologische Kompensationsfähigkeit des Körpers hinausgehen und zu gesundheitlichen Beeinträchtigungen oder Schädigungen führen. Die Tatsache, dass seitens der Öffentlichkeit und der Medien häufig nicht zwischen diesen beiden Termini unterschieden wird und ein biologischer Effekt als eine negative gesundheitliche Auswirkung interpretiert wird, führt oft zu Verwirrungen.

2) Häufig kapitulieren Interessierte vor einem unüberschaubaren Informationsangebot. Orientierung können etliche Datenbanken geben. Einen Überblick über den Stand der EMF-Forschung liefert die Literaturdatenbank der Weltgesundheitsorganisation (http://www.who.int/peh-emf/resear $\mathrm{ch} /$ database/en/index.html). Sehr umfassend ist das EMF-Portal des Forschungszentrums für Elektro-Magnetische Umweltverträglichkeit an der Rheinisch-Westfälischen Technischen Hochschule Aachen (http://www.emf-portal.de), das sich auch an Laien wendet. Das Portal umfasst über 10.000 begutachtete Artikel aus ca. 1.300 wissenschaftlichen Fachzeitschriften. Daneben bieten auch medizinische Literaturdatenbanken wie Medline (http://www.medline.de) und PubMed (http://www.pubmed.de) Literaturverweise und Informationen.
3) Darunter werden Schwellenwerte verstanden, unterhalb derer keine biologischen Wirkungen auftreten.

4) Entscheidend ist die vom Körper aufgenommene und in Wärme umgewandelte Strahlungsenergie. Das aussagekräftigste Maß der Energieabsorption im Körper durch hochfrequente EMF ist der SAR-Wert (Specific Absorption Rate). SAR ist somit auch der wichtigste Parameter wissenschaftlicher Studien zur Exposition mit hochfrequenten EMF. Für Mobiltelefone ist nach der Produktnorm EN 50360 europaweit ein SARWert von max. 2 Watt pro Kilogramm Körpergewicht zulässig. Die SAR-Werte aktueller Mobiltelefone liegen zwischen ca. 0,1 und 1,8 W/kg.

5) Vgl. dazu ABL.EG Nr. L 199.

6) In Deutschland wurde die grundlegende Idee des Vorsorgeprinzips im Rahmen der Umweltpolitik entwickelt, wobei dem Vorsorgeprinzip zumeist die Zielsetzungen Gefahrenabwehr, Risikovorsorge und Zukunftsvorsorge zugeordnet werden. Ebenso spielt Vorsorge im EU-Recht eine wichtige Rolle; entsprechend den Grundzügen des EU-Rechts und den Direktiven der Kommission handelt es sich beim Vorsorgeprinzip um einen allgemeingültigen Grundsatz. Dieser ist ,in konkreten Fällen anwendbar, in denen die wissenschaftlichen Beweise nicht ausreichen, keine eindeutigen Schlüsse zulassen oder unklar sind, in denen jedoch auf Grund einer vorläufigen und objektiven wissenschaftlichen Risikobewertung begründeter Anlass zu der Besorgnis besteht, dass gefährliche Folgen für die Umwelt und Gesundheit von Menschen, Tieren und Pflanzen mit dem hohen Schutzniveau der Gemeinschaft unvereinbar sein könnten “ (Kommission der Europäischen Gemeinschaften 2000, S. 10). Gesundheitliche und ökologische Aspekte bei mobiler Telekommunikation und Sendeanlagen können nach dieser Definition die Anwendung des Vorsorgeprinzips rechtfertigen.

7) Von Relevanz ist hier insbesondere das umfassende und bis 2009 laufende internationale EMFProjekt der WHO (http://www.who.int/emf) sowie das ebenfalls umfangreich angelegte und 2001 gestartete britische Mobilfunk-Forschungsprogramm MTHR (Mobile Telecommunications and Health Research Programme; http://www.mthr.org.uk/ind ex.htm). Ende 2007 wurde ein Zwischenbericht vorgelegt, von vielen der Projekte wurden Ergebnisse in wissenschaftlichen Publikationen veröffentlicht (http://www.mthr.org.uk/research_projec ts/research.htm). 


\section{Literatur}

Baumgärtner, N., 2005: Risiko- und Krisenkommunikation. Rahmenbedingungen, Herausforderungen und Erfolgsfaktoren - dargestellt am Beispiel der chemischen Industrie. München

Bechmann, G.; Stehr, N., 2000: Risikokommunikation und die Risiken der Kommunikation wissenschaftlichen Wissens: Zum gesellschaftlichen Umgang mit Nichtwissen. In: GAIA 9/2 (2000), S. 113-121

Bentele, G.; Brosius, H.-B.; Jarren, O. (Hg.), 2003: Öffentliche Kommunikation. Wiesbaden

BfS - Bundesamt für Strahlenschutz, 2008: Ergebnisse des Deutschen Mobilfunk Forschungsprogramms (DMF). Bewertung der gesundheitlichen Risiken des Mobilfunks (Stand 15.5.2008), Salzgitter (Bd. BfSSG-08/08; Fachbereich Strahlenschutz und Gesundheit im Bundesamt für Strahlenschutz, Juni 2008)

Dîfu - Deutsches Institut für Urbanistik, 2008: Jahresgutachten 2007 zur Umsetzung der Zusagen der Selbstverpflichtung der Mobilfunkbetreiber. Berlin

FDA - Food and Drug Administration (Hg.), 2008: Identification of Research Needs Relating to Potential Biological or Adverse Health Effects of Wireless Communication Devices. NAS Report, Washington

Kommission der Europäischen Gemeinschaften (Hg.), 2000: Die Anwendbarkeit des Vorsorgeprinzips. Mitteilungen der Kommission, COM 2000(1), Brüssel, 2. Februar 2000

Luhmann, N., 1989: Vertrauen. Ein Mechanismus der Reduktion sozialer Komplexität. Stuttgart

Meyer, A., 2008: Grüne Welle? In: c’t 15 (2008), S. $80-83$

Petermann, Th., 2001: Technikkontroversen und Risikokommunikation. In: TAB-Brief 20 (2001), S. 5-20

Renn, O.; Schweizer, P.-J.; Dreyer, M. et al., 2007: Risiko. Über den gesellschaftlichen Umgang mit Unsicherheit. München

Revermann, Chr., 2003: Risiko Mobilfunk. Wissenschaftlicher Diskurs, öffentliche Debatte und politische Rahmenbedingungen. Berlin

Schütz, H., 2008: Risikokommunikation - eine Sache des Vertrauens? In: Forschungsgemeinschaft Funk e.V. - Newsletter 16/1 (2008), S. 10-19

Siegrist, M.; Earle, T.C.; Gutscher, H. et al., 2005: Perception of Mobile Phone and Base Station Risks. In: Risk Analysis 25/5 (2005), S. 1253-1264

Siegrist, M.; Keller, C.; Cousin, M.-E., 2006: Implicit Attitudes Toward Nuclear Power and Mobile Phone Base Stations: Support for the Affect Heuristic. In: Risk Analysis 26/4 (2006), S. 1021-1029
WHO - World Health Organization (Hg.), 2006: WHO Research Agenda for Radio Frequency Fields. http://www.who.int/peh-emf/research/rf_research_ agenda_2006.pdf (download 13.12.08)

Wiedemann, P.M., 2008: Was tun mit dem Restrisiko? Risikowahrnehmung und Risikokommunikation. In: BG Elektro Textil Feinmechanik, Forschungsgemeinschaft Funk e.V. (Hg.): Gepulste Felder - eine besondere Gefahr für die Gesundheit? (Monographie) Köln, Bonn, S. 143-147

Wiedemann, P.M.; Clauberg, M.; Schütz, H., 2003: Understanding Amplification of Complex Risk Issues: The Risk Story Model Applied to the EMF Case. In: Pidgeon, N. et al. (Hg.): The social amplification of risk. New York, S. 286-301

Wiedemann, P.M.; Schütz, H., 2002: Wer fürchtet den Mobilfunk? Gruppenspezifische Differenzen bei der Risikowahrnehmung. Forschungszentrum Jülich, Programmgruppe MUT, Jülich; http://www.fz-juelich.de/mut/hefte/heft_84.pdf (download 13.12.08)

Wiedemann, P.M.; Schütz, H., 2003: Risikokommunikation: Mobilfunk und Gesundheit. Publikation des Forschungszentrums Jülich. Jülich

Wiedemann, P.M.; Schütz, H., 2008: Risikokommunikation als Aufklärung: Informieren über und Erklären von Risiken. In: Lantermann, E.D.; Linneweber, V. (Hg.): Enzyklopädie der Psychologie (Vol. 2), Serie IX: Umweltpsychologie - Bd. 1. Göttingen (im Erscheinen)

\section{Kontakt}

Dr. Christoph Revermann Büro für Technikfolgen-Abschätzung beim Deutschen Bundestag (TAB) Neue Schönhauser Str. 10, 10178 Berlin Tel.: +49 (0) 30 / 28491 - 109 E-Mail: revermann@tab.fzk.de 